\title{
Prevention of Burst Abdomen By Interrupted Closure: A Comparative Study of Conventional Continuous Versus Interrupted-X-Type Versus Hughes Far-And-Near Interrupted Abdominal Fascial Closure in Surgical Patients
}

\author{
Dr. Kuldip Singh Ahi ${ }^{*}$,Dr. Sachin M. Khandekar ${ }^{*}$,Dr. Sushil Kumar Mittal", \\ Dr. Vikram Chaudhary ${ }^{* *}$ Dr. Ankur Sharma ${ }^{@}$,Dr Aditya Jain ${ }^{1}$, \\ Dr Avnish Kumar ${ }^{2}$ \\ *Associate Professor, Department Of General Surgery, ${ }^{* *}$ Resident, Department Of General Surgery, ${ }^{*}$ Professor, \\ Senior Resident ${ }^{\circledR}$ Department Of General Surgery, Government Medical College, Patiala, Punjab, India. ${ }^{I}$ \\ Assistant Professor, Department Of Physiology, ${ }^{2}$ Professor, Department Of Physiology, Government Medical \\ College, Patiala, Punjab, India.
}

\begin{abstract}
Background: The occurrence of sudden disruption of the abdominal laparotomy wound is a major disaster in the life of a patient who has undergone an abdominal operation and a major psychological blow to the patient as well as the surgeon.Its prevention is important in reducing post-operative morbidity and mortality.The search for the optimal laparotomy technique has gone on for more than 100 years and will continue.

Aims: The present study was undertaken to assess the incidence and relative risk of burst abdomen in post midline laparotomypatient using continuous suture versus interrupted X suture versusHughes Far-and-Near technique in sheath closure.

Materials and Methods: This was a prospective randomised study. The data were collected at surgical wards of Rajindra Hospital, Government Medical College, Patiala. 90 patients undergoing emergency laparotomy through midline vertical incisions were randomized after informed consent by either continuous closure or by interrupted X or by Hughes Far-and-Neartechnique. The major outcome variable is risk of burst abdomen diagnosed by a consultant. The risk of burst in each group and relative risk (RR) of burst (using continuous group as the reference category) were assessed

Main Outcome Variable: The risk of burst abdomen diagnosed by a consultant.

Predictor Variables: wound infection, abdominal distension, cough, diabetes, anaemia, uraemia, malnutrition, old age, steroid intake, duration of surgery, duration of illness, smoking, alcohol, ileostomy, leakage of primary repair, early ambulation.

Statistical Analysis: The risk of burst (incidence) in each group and relative risk (RR) of burst (using continuous group as the reference category) were calculated.

Result: Ninety patients undergoing emergency laparotomy were randomized into three arms to undergo closure with continuous, interrupted-X, and Hughes Far-and-Near suturing techniques. Burst abdomen occurring up to 4 weeks of operation. Nineteen (21.1\%) of 90 patients developed burst in the post-operative period. 11(36.7\%) of 30 patients in continuous arm developed burst. Four of 30 (13.3\%) patients in Interrupted-X arm and 4 of 30 (13.3\%) patients in Hughes Far-and-Near arm developed burst. The relative risk for burst abdomen with continuous method as reference category and interrupted method as exposure category was $1.868(p=0.011)$.

Conclusion: Interrupted-X suture and Hughes Far-and-Near Interrupted Methods of suturing better than the Conventional Continuous methods in the management of closure of vertical laparotomy incision as the postoperative complications like wound dehiscence and wound infection were found to be significantly lesser with the use of interrupted method of closure. Wound infection (SSI), old age (>60 years), steroid intake, cough, duration of surgery, ileostomy, leakage of repair, and intake of alcohol are significant risk factors for burst abdomen.
\end{abstract}

Keywords: Abdominal wound dehiscence, Burst abdomen, Suture technique, Randomized trial, Emergency laparotomy, Prof Hughes' Far-and-Near suture.

\section{Introduction}

Abdominal wound dehiscence or burst abdomen is disruption of any or all off the layers in a wound.Abdominal wound dehiscence is a common complication of emergency laparotomy in cases of perforation peritonitis in Indian setup. ${ }^{[1]}$ As majority of our patients are from rural background, with poor nutritional status due to poverty and some are having addiction like tobacco chewing, alcohol consumption and 
smoking. Most of time, they get neglected for the initial part of their illness due to poor access to better health care facilities, unless they are shifted to higher centre. Because of delay in the management of a case of perforation there is continuous increase in the septic load of abdominal cavity, significant third space loss, dehydration, deranged renal function, multisystem organ failure and septicaemia. All of these lead to increase in morbidity in form of wound infection and burst abdomen and even death. Wound dehiscence most commonly occurs from the $5^{\text {th }}$ to the $8^{\text {th }}$ postoperative day when the strength of the wound is at its weakest. ${ }^{[2]}$ The incidence of dehiscence ranges from $0 \%$ to $6 \%^{[3-14]}$ in different patient series and the associated mortality rate ranges from $10 \%$ to $35 \%{ }^{[15]}$ in most of the centres across the world, some centres in India have recorded an incidence rate of burst abdomen as high as $10-30 \% .^{16,17}$

There have been a number of studies evaluating various closure techniques ${ }^{[18]}$ and suture materials. Studies carried out in the West have found nosignificant difference in the risk of burst between continuous and interrupted methods ${ }^{[19]}$. The choice of method of closure may not be very crucial in patients undergoing elective laparotomywith adequate enutritionalstatusandnootherriskfactor for burst and are well prepared for surgery, but in developing countries such as India, most patientspresentin emergency setting withoneormoreriskfactorssuchasprolonged intraperitoneal sepsis and malnutrition. Hence, it is imperative for us to ascertain the safest method of closing the abdomen. This present study was undertaken to compare in a prospective randomized fashion, to assess the proportion of Burst abdomen in post midline laparotomy patients undergoing Conventional Continuous versus Interrupted-X suture versus Hughes Far-and-Near Interrupted Methods of midline abdominal wound closure.

\section{Aims And Objectives}

1. To evaluate the incidence of Abdominal Wound Dehiscence or Burst Abdomen with:

(a) Continuous suturing method

(b) Interrupted-X suturing method,

(c) Hughes Far-and-Near Interrupted Methods.

2. To compare the relative risk of Abdominal Wound Dehiscence or Burst Abdomen between Continuous, Interrupted- $\mathrm{X}$ and Hughes Far-and-Near interrupted type abdominal fascial closure

\section{Material And Methods}

The present prospective comparative randomized study was carried out on total of 90 patients presenting to the Casualty Department of General Surgery, Rajindra Hospital, Patiala undergoing emergency laparotomy. The study Design was Double-blinded as the patient and the surgeon evaluating the outcome were blinded about the method of suturing.

90 patients undergoing emergency laparotomy for acute abdominal obstruction, peritonitis, and abdominal trauma, were included after getting an informed consent. Patients having a previous laparotomy or burst abdomen or incisional hernia at presentation were excluded.

These patients were randomized to divide into three equal groups with 30 each:

Group A - Conventional Continuous Suture

Group B - Interrupted-X Sutures

Group C- Hughes Far-and-Near Interrupted Sutures

\section{Inclusion Criteria}

1. All patients undergoing emergency laparotomy by midline incision.

2. All patients giving written informed consent for enrolment in the study.

\section{Exclusion Criteria}

1. Patients are younger than 18 years of age

2. Patients who had undergone a previous laparotomy for any condition (or had an incisional hernia or burst abdomen at presentation)

3. Patients who refused to participate in study

All patients were explained about the study and its design and a written consent was obtained. They were randomized to undergo either Continuous or Interrupted-X or Hughes Far-and-Near interrupted closure of the laparotomy incision using simple random sampling. A detailed history and clinical examination was conducted in all the patients who presented to surgery emergency with provisional diagnosis of perforation peritonitis after taking detailed consent. The investigator was personally involved in all the cases preoperatively, during the surgery and for the post-operative care. Data about the onset of symptoms, duration of symptoms, any treatment taken, history of any chronic illness in form of diabetes, hypertension, pulmonary tuberculosis, history of addiction and history blunt trauma abdomen, was taken and noted on proforma. 
Patients were thoroughly examined, vitals recorded, patients checked for the presence of pallor, icterus, cyanosis, clubbing, generalized lymphadenopathy and oedema feet. Abdominal examination was done in detailed manner and checked for the presence of guarding, rigidity, distension obliterated liver dullness or any scar from previous surgery. Respiratory and cardiovascular system was also evaluated.Time delay between onset of symptoms and surgery in form of duration of illness was also recorded.

Routine investigations in the form of haemoglobin, total leucocyte count, differential leucocyte count, blood sugar levels, renal function tests. Serum electrolyte levels and urine complete examination was done.Radiological investigations done were plain X-ray chest with both dome of diaphragm. X-ray abdomen (erect and supine position) and ultrasound abdomen were done wherever indicated.After resuscitation with intravenous fluids along with Foley's catheterization and nasogastric tube in situ patients were posted for emergency laparotomy. Under general anaesthesia, all the patients were opened through a midline abdominal incision and the surgical procedure was conducted according to the requirement of the underlying disease. After dealing with the primary pathology, a through peritoneal lavage was performed in all cases. Two drains were placed in the peritoneal cavity one in Morison pouch and one in pelvic cavity and brought out through separate stab incisions. The closure of midline incision was carried out accordingly. Time required for completion of suturing noted. In hospital morbidity in the form of burst abdomen, wound infections, ileostomy, leakage of repair and re-exploration was taken as final outcome.

\section{Suturing Techniques}

The methods of midline abdominal incisional closure are as follows.

(a) Continuous Closure: It was performed using No. 1 Prolene suture (polypropylene), care being taken to place each bite $1-1.5 \mathrm{~cm}$ from the cut edge of linea alba and successive bites being taken $1 \mathrm{~cm}$ away from each other (Fig.1). The edges of lineaalba were gently approximated without strangulation with an attempt to keep a suture to wound length ratio of $4: 1 .^{[1]}$

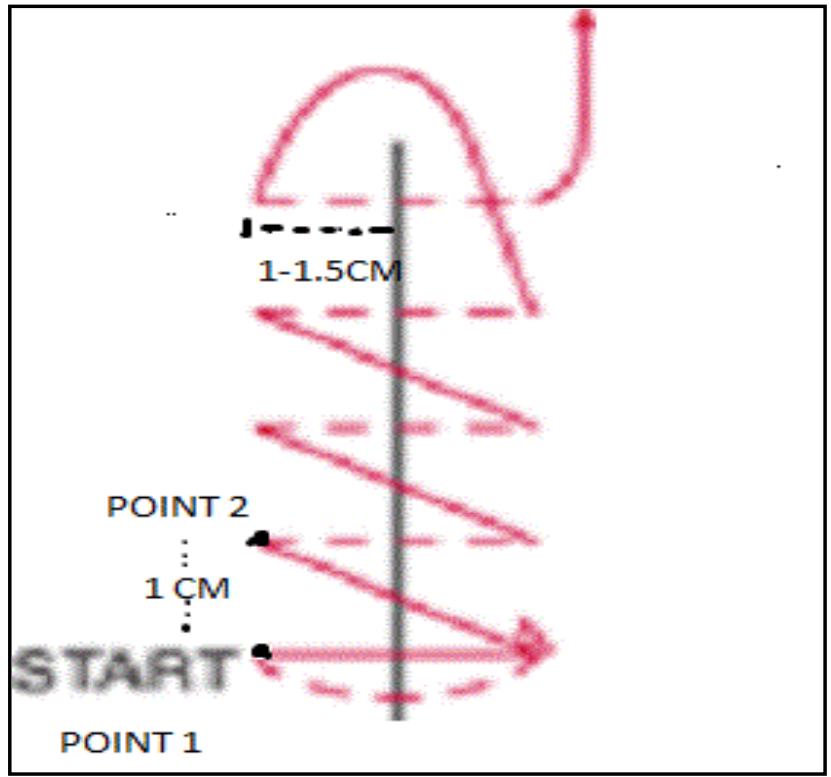

Figure 1: Continuous Suture

(b) Interrupted Double-X Closure: It was performed using No. 1 Prolenesuture (polypropylene). A large bite was taken on the cut edge of lineaalba at point (1) from outside-in, $2 \mathrm{~cm}$ from edge. The needle emerged on the other side from point (2) inside-out diagonally $2 \mathrm{~cm}$ from the edge and $4 \mathrm{~cm}$ above or below the first bite. This strand was crossed or looped around the free end of suture and continued outside-in, at point (3) and comes at point (4), diagonally at 90 degree to the first diagonal. The two ends were tied just tight enough to approximate the edges of linea alba, taking care not to include bowel or greater omentum between the edges. The small free end of the suture is passed deep to the $\mathrm{X}$ behind linea alba and again tied to the other end of the suture. This method of tying four throws in front and four throws behind the $\mathrm{X}$ created two X-like crosses - one on the surface and another deep to linea alba. The central knot allowed fixation of four arms of the $\mathrm{X}$ like a pivot. The next $\mathrm{X}$-suture was placed $1 \mathrm{~cm}$ away (above or below) from the previous one. Thus, in a $14 \mathrm{~cm}$ long wound, $3 \mathrm{X}$-sutures were applied (Fig. 2). The suture line was then palpated for any gap with the index finger. Any large gap permitting a finger was closed with a simple interrupted suture. 


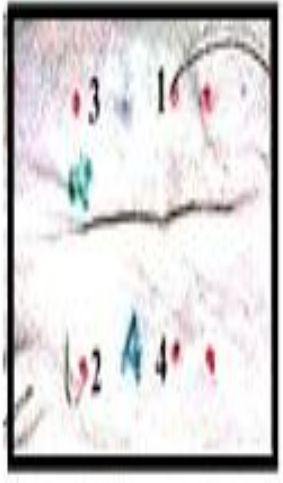

(a)

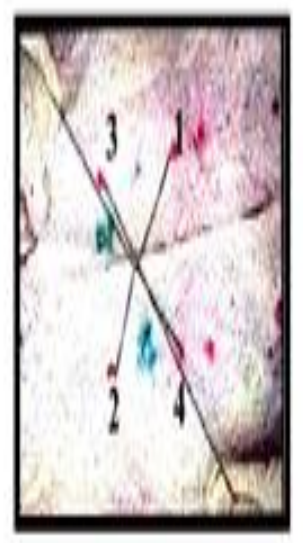

(d)

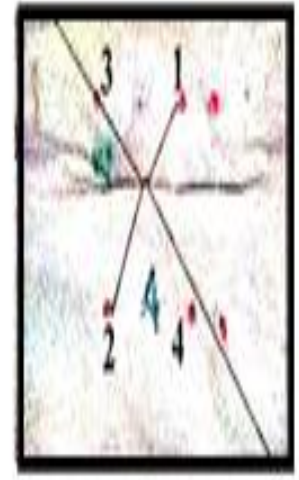

(b)

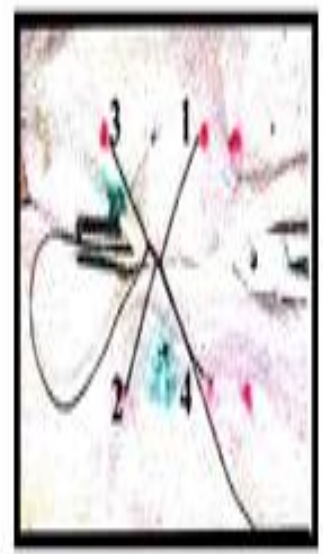

(e)

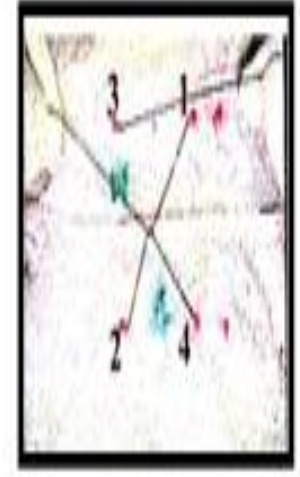

(C)

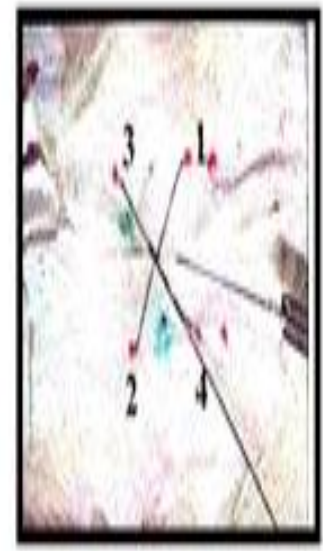

(I)

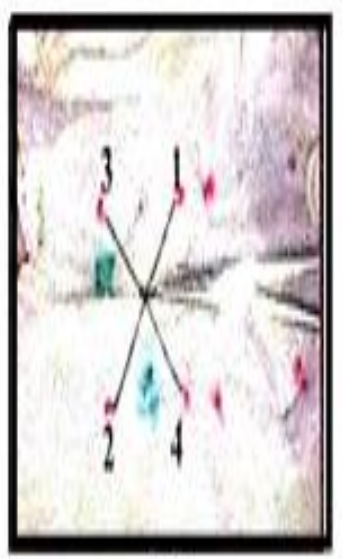

(a)

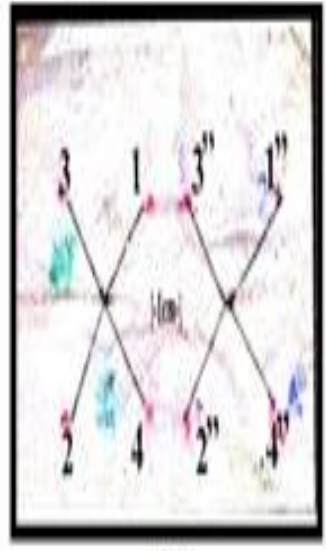

(h)

Figure 2: Interrupted X-Suture

(c) Prof. Hughes Far-and-Near Interrupted Method: The patients in the third arm were treated by Professor Hughes modification of Smead-Jones far-and-near stitch (Fig.3). This comprised a far bite starting at point 
(1) $2 \mathrm{~cm}$ on the edge of linea from outside-in and then taking a near bite of $0.5 \mathrm{~cm}$ at point (2) on the other side inside-out a near bite on the same side outside-in at point (3) and then a far bite on the other side inside-out at point (4). The suture was next converted to a horizontal mattress by taking a far bite $1 \mathrm{~cm}$ above or below the previous bite on the other side at point (5) outside-in near bite on the same side at point (6) inside-out, near bite on the other side at point (7) outside-in, and finally a far bite on the same side at point (8) inside-out. The two ends of the suture were tied to approximate the edges of the linea alba.

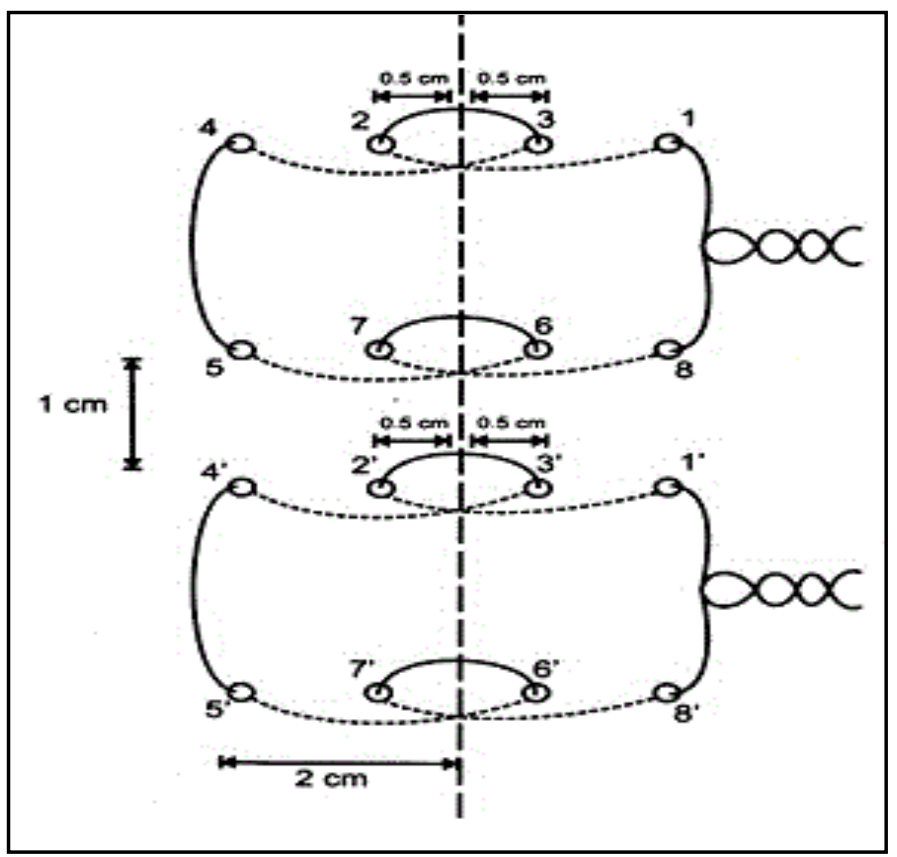

Figure 3: Far and near double horizontal mattress suture developed by Prof. L.E. Hughes at Cardif

\section{Follow-up}

Each incision was specifically followed and investigated for hematoma, infection, any discharge from wound, dehiscence or burst abdomen. The wound was managed by daily antiseptic dressings and intravenous antibiotics. The patients who developed wound infection, twice daily change in dressing done. Any discharge from wound sent for culture and sensitivity and accordingly intravenous injectable were started. Each patient was followed up on $1^{\text {st }}$ week, $2^{\text {nd }}$ week than on $4^{\text {th }}$ week after surgery to determine the risk of Abdominal wound dehiscence or burst abdomen.

\section{Measurement Of Variables}

The main outcome variable was presence of an abdominal wound dehiscence or burst. A burst was considered present when intestine, omentum, or other viscera were seen in the abdominal wound up to 30 days from the date of operation. A consultant surgeon ascertained the occurrence of burst. This was recorded as a binary variable-present/absent.

\section{The following predictor variables were also recorded:}

- Intra-peritoneal sepsis-coded as a binary variable, present/absent.

- Coughing-present/absent.

- Diabetes-coded as present/absent. Its presence was defined as fasting blood sugar $>140 \mathrm{mg} / \mathrm{dl}$ or random blood sugar $>200 \mathrm{mg} / \mathrm{dl}$.

- Uraemia- coded as a binary variable, present or absent. Its presence was defined as blood urea greater than $50 \mathrm{mg} / \mathrm{dl}$.

- Abdominal distension- coded as a binary variable, present/absent.

- Malnutrition-coded as binary variable, present/absent. Its presence was defined as weight $<70 \%$ of expected weight.

- Anaemia-coded as binary variable, present/absent. Its presence was defined as haemoglobin less than 10 $\mathrm{g} / \mathrm{dl}$.

- Steroid intake-coded as a binary variable, present/ absent. If present, dose and duration of treatment was noted.

- Illeostomy - coded as a binary variable, present/ absent.

- Early ambulation - coded as a binary variable, present/ absent.

DOI: 10.9790/0853-1602072130 www.iosrjournals.org 25 | Page 
- Smoking - coded as a binary variable, present/ absent.

- Alcohol - coded as a binary variable, present/ absent.

- Duration of Surgery - coded as a binary variable, long $>2$ hours/ short $\leq 2$ hours.

- Duration of illness - coded as a binary variable, $<3$ days/ $\geq 3$ days.

Data was entered and statistical analysis was done.

\section{Limitations of study:}

The follow up period is short and so incisional hernia was not taken into consideration.

\section{Statistical analysis:}

The following statistical analyses were carried out:

1. Risk of burst: The risk (cumulative incidence) of burst was calculated as the number of burst abdomen patients in a group/total number of patients in that group. The point estimate and $95 \%$ confidence intervals were calculated using STATA 10 software package (STATA Corporation,Texas). Fisher's exact test or Mantel-Haenszel chi-square test was used for hypothesis testing. Two-tailed P values were used with $\alpha$ or type one error set at 0.05 .

2. Relative risk of burst abdomen: The risk of dehiscence was similar with the two types of interrupted sutures (X and Prof Hughes far and near). Hence, the data of these groups were added together and named "interrupted suture" group. The relative risk of this interrupted group as the "exposure" was calculated against the continuous method as the "reference" category.

\section{Results}

A total of 90 patients satisfying inclusion criteria were included in the study carried out at Rajindra hospital, Government medical college, Patiala.

Age Distribution: The mean age of the patients was 42.08 years $(\mathrm{SD}=15.754)$ with a median of 34.89 years. The age ranged from 18 to 83 years.

Sex Distribution: In the present study majority of patients were males $(84.4 \%)$ and females were only $(15.6 \%)$. Group A included 27 (35.5\%) males and $3(21.4 \%)$ females, Group B included $24(31.6 \%)$ males and $6(42.9 \%)$ females whereas Group C included 25 (32.9\%) males and 5 (35.7\%) females as study subjects. Male to female ratio was 5.4:1.

Rate of Burst Abdomen: There were total 19 patients, who developed burst abdomen in postoperative period. Maximum number of burst abdomen occurred on $8^{\text {th }}(8 / 19,8.9 \%)$ and $10^{\text {th }}(4 / 19,4.4 \%)$ day and $(3 / 19,3.3 \%) 9^{\text {th }}$ day post operatively. In present study the rate of burst abdomen was found to be $21.1 \%$.

In present study the rate of burst abdomen was found to be $36.7 \%$ in group A (continuous sutures) and $13.3 \%$ in group B (interrupted X suture), and 13.3\% in Group C (Hughes Far-and-Near suture) (p =0.038).

Our study described significance difference between continuous and interrupted method for the development of burst abdomen. ( $\mathrm{p}=0.038)$.

There was no statistically significant difference in the risk of burst abdomen between the Group B (Interrupted-X suture) and Group C (Hughes Far-and-Near suture). Since these methods are two types of interrupted closure techniques, they were grouped under "interrupted method" for relative risk (RR) calculation as given below.

Risk Estimate for burst abdomen with continuous method as reference category and interrupted method as exposure category was 3.763 (Odds Ratio for Group A/B and $\mathrm{C}=3.763$ ) (95\% CI 1.315-10.769; $\mathrm{p}=0.011$ ). It means that there are nearly 3.7 times chances of burst abdomen with continuous suture. The relative risk for burst abdomen with continuous method as reference category and interrupted method as exposure category was 1.868 .

\section{Age and Burst Abdomen:}

The rate of burst abdomen was found to be $40.00 \%$ (6/15) in old patients (>60 years) as compared to young patients $17.30 \%(13 / 75)(\mathrm{p}=0.05)$. The relative risk $(\mathrm{RR})$ of development of burst abdomen in older age patients was found to be 2.309 .

\section{Anaemia and Burst Abdomen:}

In present study, 25 patients had $\mathrm{Hb}<10 \mathrm{gm} \%$ who were labelled as anaemic patients. Remaining 65 non anaemic patients marked as control having $\mathrm{Hb} \geq 10 \mathrm{gm} \%$. Out of 25 anaemic patients $8(32 \%)$ developed burst abdomen compared to 11 out of $65(16.9 \%)$ non anaemic patients. The anaemia was not found out to be a statistically significant risk factor for burst abdomen $(\mathrm{p}=0.116)$. The relative risk of burst abdomen for anaemia was found 1.891 . 


\section{Malnutrition and Burst Abdomen:}

In our study 43 patients presented with malnutrition. Out of these 43 patients, $12(27.9 \%)$ patients developed burst abdomen. Out of remaining 47 well-nourished patients 7 (14.9\%) patients developed burst abdomen. Malnutrition was not found to be a significant risk factor for burst abdomen ( $\mathrm{p}=0.131)$. Relative risk of burst abdomen for malnutrition was 1.917.

\section{Diabetes and Burst Abdomen:}

In present study out of 6 diabetic patients, 2 patients were known diabetic and 4 were diagnosed in hospital. Out of these 2 (33.3\%) patients developed burst abdomen compared to 17 (20.2\%) patients out of 84 who were non diabetic. The analysis of above table was not statistically significant $(\mathrm{p}=0.448)$ and the relative risk of burst abdomen for diabetes was 1.648 .

\section{Steroid intake and Burst Abdomen:}

In present study 8 patients out of 90 study group had history of steroid intake. Out of these 8 patients, 6 (75.0\%) patients developed burst abdomen in post-operative period compared to 13 (15.9\%) patients out of 82 patients who did not give history of steroid intake. The analysis of above table was highly statistically significant $(\mathrm{p}=0.000)$ and the relative risk of burst abdomen in patients having steroid intake was 4.721 .

\section{Uraemia and Burst Abdomen:}

In present study 20 patients had uraemia, out of these $5(25.0 \%)$ patients developed burst abdomen. Remaining 70 patients were found to be non-uremic, out of these 14 (20.0\%) patients developed burst abdomen. The analysis of above table was not statistically significant $(\mathrm{p}=0.629)$ and the relative risk of burst abdomen for uraemia was 1.25 .

\section{Duration of surgery and Burst Abdomen:}

There were 28 patients in whom duration of surgery was long (>2hrs) out of 90 study group. Out of these 28 patients, $12(42.9 \%)$ developed burst abdomen in post-operative period compared to $7(11.3 \%)$ patients out of 62 patients in whom duration of surgery was short $(<2 \mathrm{hrs})$. The analysis of above table was highly statistically significant $(\mathrm{p}=0.001)$ and the relative risk of burst abdomen in patients having long duration of surgery was 3.794 .

\section{Cough and Burst Abdomen:}

In present study 17 patients had cough and pulmonary infection out of these $12(70.6 \%)$ patients developed burst abdomen compared to 7 (9.6\%) patients out of 73 who did not have cough or pulmonary complications. The analysis of above table was statistically highly significant ( $\mathrm{p} 0.000)$ and the relative risk of burst abdomen for cough was found 7.356.

\section{Duration of Illness and Burst Abdomen:}

As shown in above table, maximum number of patients 59 presented to hospital and underwent surgery within 3 days of developing symptoms out of these 11 (18.6\%) develop wound dehiscence/burst. 31 patients presented with more than 3 days and $8(25.8 \%)$ developed wound dehiscence/burst. The analysis of above table was statistically not significant (p 0.429) and the relative risk of burst abdomen for duration of illness was found 1.597 .

\section{Smoking and Burst Abdomen:}

In present study 31 patients were cigarette/bidi smoker compared to remaining 59 patients who were non-smoker. Out of these 31 smoker patients 9 (29.0\%) developed burst abdomen compared to 10 (16.9\%) patients out of 59 non-smoker patients. The analysis of above table was not statistically significant $(\mathrm{p}=0.182)$ and the relative risk of wound infection for smoking was 1.714 .

\section{Alcohol intake and Burst Abdomen:}

In present study 22 patients were known alcoholic compared to 68 non-alcoholic patients out of 90 study group. Out of these 22 patients, 8 (36.4\%) had burst abdomen in post-operative period compared to 11 $(16.2 \%)$ out of 68 non-alcoholic patients. The analysis of above table was statistically significant $(\mathrm{p}=0.044)$ and the relative risk of wound infection for alcoholic patients was 2.247.

\section{Ileostomy and Burst Abdomen:}


Out of total 90 patients, 19 patients underwent ileostomy. Out of these 19 patients, $10(52.6 \%)$ patients developed burst abdomen in post-operative period compared to $9(12.7 \%)$ patients out of 71 patients in whom ileostomy not done. The analysis of above table was highly statistically significant $(\mathrm{p}=0.000)$ and the relative risk of burst abdomen in patients having ileostomy was 4.148.

\section{Leakage of primary repair and Burst Abdomen:}

According to above table only 2 patients developed leakage of primary repair of intra-abdominal pathology in post-operative period. These two patients (100\%) had wound infection and subsequently developed wound dehiscence/burst abdomen in post-operative period. Out of remaining 88 patients 17 (19.3\%) patients had wound infection in post-operative period. The analysis of above table was highly statistically significant $(\mathrm{p}=0.006)$ and the relative risk of wound infection in patients having leakage of primary repair was 4.301 .

\section{Wound infection (SSI=surgical site infection) and Burst Abdomen:}

In present study 29 patients developed wound infection in post-operative period out of 90 study group. Out of these 29 patients, 19 (65.5\%) patients developed burst abdomen.10 (34.5\%) patients underwent wound healing without development of burst abdomen. 61 patients out of 90 study group who did not have wound infection underwent normal wound healing. No patient developed burst abdomen who did not have wound infection. The analysis of above table was highly statistically significant $(\mathrm{p}=0.000)$ and the relative risk of burst abdomen in patients having wound infection was 75.777 .

\section{Discussion}

The discussion on the subject of abdominal wound dehiscence/Burst abdomen is as old as the history of modern operative surgery. The perioperative mortality and long term morbidity associated with the condition need medical surgical preventive measures to be taken. Abdominal wound dehiscence is a devastating incident that can cause pain, mentaldistress, infectious complications, and financial burdens forthe patient, as well as complications including eviscerationand reoperation ${ }^{[5,14,21]}$. Surgeon expertise, type of incision, suturing material, surgical site infection, nutritional status, persistent cough, abdominal distension, leakage of pancreatic enzyme, anemia, obesity, diabetes, jaundice, old age, emergent operation, particular procedures such as colon surgery, and late wound healing due to malignancy have all been suggested to predispose patients to abdominal wound dehiscence.Some of these factors are unavoidable ${ }^{[22-24,]}$. Different surgical techniques for closing the wound should be carefully considered ${ }^{[25]}$. Suture materials are of great importance in providing sufficient strength and influencing adverse events ${ }^{[6]}$.

In my study, it was found that those undergoing emergency laparotomy having multiple risk factors adverse to wound healing suffered from a burst in $21.1 \%$ of cases. Indian authors have reported burst abdomen to occur in $10-30 \%$ of emergency cases. ${ }^{[16,17,26]}$ Richards $^{[27]}$ et al. in their randomized prospective study of 571 patients comparing continuous versus interrupted suture technique for abdominal fascial closure came out with following results. In midline incision, dehiscence rate was $2.0 \%$ (5/244) for the continuous group versus $0.9 \%$ $(2 / 229)$ for the interrupted group. The difference was not statistically significant $(\mathrm{p}=0.19)$. Fagniez ${ }^{[28]}$ et al. in their study titled "abdominal midline incision closure." A multi-centric randomized prospective trial of 3135 patients, comparing continuous versus interrupted polyglycolic acid suture came out with following findings. The overall dehiscence rate was $1.6 \%$ in continuous suture group versus $2 \%$ in the interrupted suture group.

A study conducted in 2004 by Srivastava et $\mathbf{a l}^{[18]}$ in surgical wards of All India Institute of Medical Science (AIIMS), on a total of 100 patients undergoing emergency laparotomy and 110 patients undergoing elective laparotomy through a midline vertical incision were randomized to either a continuous closer or an interrupted $\mathrm{X}$ technique. The risk of burst abdomen was diagnosed by a consultant. The risk of burst in each group and relative risk (RR) of burst (using continuous group as the reference category) were calculated. There was one burst (out of 46) in the X suture group and 8 bursts (out of 54) in the continuous arm in the emergency group. The RR for the burst (continuous group as the reference category) was 0.15 (95\% CI : 0.02 to 1.13 ( $\mathrm{p}=0.028$ ). They concluded that dehiscence can be reduced using interrupted X-closure. Intraperitoneal sepsis is the single most important factor in predicting burst abdomen. Multivariate Logistic Regression revealed method of suturing, cough, sepsis, anaemia, malnutrition and abdominal distension to be significant predictors of burst abdomen.

Agrawal et $\mathbf{a l}^{[1]}$ (2014) studied reduction in the risk of abdominal dehiscence with application of interrupted method of laparotomy closure and comparison with risk of burst with continuous method of closure. Three hundred forty eight patients undergoing laparotomy (114-elective gynaecology, 114-emergency gynaecology, 120-emergency surgery) were randomized into three arms to undergo closure with continuous, interrupted-X, and Modified Smead-Jones suturing techniques. Burst abdomen occurring up to 4 weeks of operation. Twenty-nine $(8.33 \%)$ of 348 patients developed burst in the post-operative period. $19(15.70 \%)$ of 121 patients in continuous arm developed burst. 5 of $110(4.55 \%)$ patients in Interrupted-X arm and 5 of 117 
(4.27\%) patients in Modified Smead-Jones arm developed burst. Interrupted suturing was associated with significant reduction in risk ofburst when compared with continuous closure. Important predictors of burst were intraperitoneal sepsis, cough, uraemia, and surgical site infection. They concluded that in presence of these symptoms, the risk of abdominal wound dehiscence can be reduced to less than one-third by using interrupted sutures. Continuous suture gives good results in elective setting. Jaundice, anaemia, malnutrition, and intraabdominal malignancy do not increase the risk of burst.

From above discussion, it is evident that most of the clinical trials from western centres found no obvious difference in wound dehiscence in midline laparotomy between interrupted and continuous closure techniques. However, clinical trials from Indian centres report less number of dehiscence with interrupted fascial closure. This may be because in India patients undergoing emergency laparotomy have poor clinical profile at the time of presentation. In a continuous suturing cutting out of even a single bite of tissues, leads to opening of the entire wound. This is the probable explanation for the high prevalence of burst in continuous suturing group of emergency laparotomy.

In present study, a statistically significant difference in the risk of burst was obtained between the continuous and interrupted arms. However, there was no significant difference in the risk of burst between the two types of interrupted methods, namely interrupted X and Prof Hughes far-and-near suture. In presence of certain factors (e.g., old age, cough, uraemia, ileostomy, duration of surgery, steroid intake, leakage of primary repair, alcohol consumption and wound infection), the risk of burst increases manifold. It is in these cases that we need to apply the suturing method mosteffective in preventing burst. The occurrence of burst increases risk of chest infection due to inability to generate positive intra-abdominal pressure and expectorate the secretions. It also increases wound pain, duration of hospital stay, and cost of care due to need for re-suturing under general anaesthesia and resources.

\section{Conclusion}

1. Interrupted-X suture and Hughes Far-and-Near Interrupted Methods of suturing better than the Conventional Continuous methods in the management of closure of vertical laparotomy incision as the post-operative complications like wound dehiscence and wound infection were found to be significantly lesser with the use of interrupted method of closure.

2. Cough, old age (>60 years), steroid intake, wound infection, duration of surgery, ileostomy, leakage of repair, method of suturing and intake of alcohol to be significantly related to increased incidence of burst abdomen.

3. Jaundice, anaemia, malnutrition, diabetes, duration of illness and smoking did not make substantial contribution to the risk of burst abdomen.

\section{Recommendation}

Interrupted suture techniques (Interrupted X or Prof. Hughes' method) should be used in all emergency laparotomy cases.

\section{Limitations Of Study}

We did not carry out long-term follow-up, and hence the risk of incisional hernia could not be calculated.

\section{References}

[1]. Agrawal CS, Tiwari P, Mishra S, Rao A, Hadke NS, Adhikari S et al. Interrupted abdominal closure prevents burst: randomized controlled trial comparing interrupted-x and conventional continuous closures in surgical and gynecological patients. Indian J Surg. 2014;76(4):270-6.

[2]. Williams NS, Bulstrode CJK, O'Connell PR. Postoperative Care. In: Bailey and Love's Short Practice of Surgery. 26thedn. Taylors and Francis Group 2013;21:279.

[3]. Webster C, Neumayer L, Smout R. Prognostic models of abdominal wound dehiscence after laparotomy. J Surg Res 2003; 109: 130-7

[4]. Wahl W, Menke H, Schnutgen M. Die Fasciendehiscenz- Ursache and Prognose. Chirurg 1992;63:666-71.1

[5]. Gislason H, Gronbech JE, Soreide O. Burst abdomen and incisional hernia after ,major gastrointestinal operations-comparison of three closure techniques. Eur J Surg1995; $161: 349-54$

[6]. Armstrong CP, Dixon JM, Duffy SW. Wound healing in obstructive. jaundice: Br J Surg $1984 ; 71$ :267-70

[7]. Penninckx FM, Poelmans S V, Kerremans RP. Abdominal wound dehiscence ingastroenterological surgery. Ann Surg1979; 189 : $345-52$

[8]. Pavlidis TE, Galatianos IN, Papaziogas BT. Complete dehiscence of thewound and incriminating factors. Eur J Surg2001; 167 : $351-4$

[9]. Humar A, Ramcharan T, Denny R. Are wound complications after a kidnev transplantmore common with modern immunosuppression? Transplantation $2001 ; 72: 1920-3$

[10]. Makela JT, Kiviniemi H, Juvonen T. Factors influencing wound dehiscence after midline laparotomy. Am J Surg 1995:; 170:387-90

[11]. Keil RH, Keitzer WF, Nicholas WK. Abdominal wound dehiscence. Arch Surg. 1973;106:573-7.

[12]. Niggebrugge AH, Hansen BE, Trimbos JB. Mechanical factors influencing the incidence of burst abdomen. Eur J Surg 1995;161:655-61.

[13]. Col C, Soran A, Col M. Can postoperative abdominal dehiscence be predicted? Tokai J ExpClin Med 1998; 23:123-7.

[14]. Riou JP, Cohen JR, Johnson H. Factors influencing wound dehiscence. Am J Surg. 1992;163:324-30.

DOI: $10.9790 / 0853-1602072130 \quad$ www.iosrjournals.org $29 \mid$ Page


[15]. Dudley HAF. Layered and mass closure of the abdominal wall.Br J Surg 1970; 57:664-67.

[16]. Singh A, Singh S, Dhaliwal US, Singh S. Technique of abdominal wall closure: a comparative study. Ind J Surg 1981;43:785 90.

[17]. Shukla HS, Kumar S, Misra MC, Naithani YP. Burst abd omen and suture material: a comparison of abdominal wound closure with monofilament nylon and chromic catgut. Indian J surg. 198 1;43:487 - 91.

[18]. Srivastava A, Roy S, Sahay KB, Seenu V, Kumar A, Chumber S, Bal S, Mehta SN (2004) Prevention of burst abdominal wound by a new technique: a randomized trial comparing continuous versus interrupted X-suture. Ind J Surg 66(1):19-27 2.

[19]. Van't Riet M, Steyerberg EW, Nellensteyn J, Bonjer HJ, Jeekel J (2002) Meta-analysis of techniques for closure of midline abdominal incisions. Br J Surg 89(11):1350-1356

[20]. Hughes LE. Incisional hernia. Asian J Surg 1990;13(2):69.

[21]. Sørensen LT, Hemmingsen U, Kallehave F, et al. Risk factors for tissue and wound complications in gastrointestinal surgery. Ann Surg 2005;241:654.

[22]. Wissing J, van Vroonhoven TJ, Schattenkerk ME, et al. Fascia closure after midline laparotomy: Results of a randomized trial. Br J Surg 1987;74:738.

[23]. Adams G, Richter RM, Levowitz BS. A safe method of closure with retention sutures. SurgGynecolObstet 1973;136:981.

[24]. Van Ramshorst GH, Salu NE, Bax NM. Risk factors for abdominal wound dehiscence in children : a case control study. World J Surg 2009;33(7):1509-13

[25]. Boissel P, Jamart J, Grumillier P, et al. A new technique for closing abdominal incisions in patients with poor wound healing. Am J Surg 1982;143:380.

[26]. 17k Chowdhury SK, Choudhury SD. Mass closure versus layer closure of abdominal wound: A prospective clinical study. J Indian Med Assoc 1994;92:229-32.

[27]. Richards PC, Balch CM, Aldrete JS. Abdominal wound closure. A randomized prospective study of 571 patients comparing continuous vs. interrupted suture techniques. Ann Surg 1983;197:238-43.

[28]. Fagniez PL, Hay JM, Lacàine F, Thomsen C. Abdominal midline incision closure. A multicentric randomized prospective trial of 3,135 patients, comparing continuous vs interrupted polyglycolic acid sutures. Arch Surg 1985;120:1351-3. 